\title{
Comparison of the Amount of Drainage in 3 Acellular Dermal Matrices in Implant-Based Breast Reconstruction: A Retrospective Study
}

\section{June-Kyu Kim, Yong Seong Kim}

Department of Plastic and Reconstructive Surgery, Kangbuk Samsung Medical Center, Sungkyunkwan University School of Medicine, Seoul, Korea
This article was presented as a poster at the 73rd Congress of the Korean Society of Plastic and Reconstructive Surgeons, on November 13-15, 2015.

No potential conflict of interest relevant to this article was reported.
Background Acellular dermal matrix (ADM)-assisted breast reconstruction has gained popularity because it has several advantages. Some studies have reported that the use of ADM was strongly associated with postoperative complications, especially seroma formation. We performed direct-to-implant breast reconstruction using 3 types of human allograft products (CGDerm ${ }^{\circledast}$, AlloDerm ${ }^{\circledast}$, and MegaDerm ${ }^{\circledast}$ ). The purpose of this study was to obtain information useful for the selection of an ADM product by comparing the amount of collected serum and the maintenance period of the drain.

Methods Seventy-three patients with breast cancer who underwent ADM-assisted implant breast reconstruction between March 2011 and January 2016 were included in this study. The patients were divided into 3 groups based on the type of ADM that was used. The demographic characteristics collected were: age, body mass index (BMI), breast reconstruction location, smoking history, axillary dissection, comorbidities (such as hypertension and diabetes mellitus [DM]), breast implant size, breast implant type, amount of ADM, the total amount of serum that was collected in the drain, and the maintenance period of the drain.

Results We obtained significant results after adjusting for variables including age, BMI, DM, axillary dissection, ADM amount, and breast implant size. Compared to AlloDerm, MegaDerm showed a significantly smaller amount of total drained serum and a significantly shorter drain maintenance period.

Conclusions The use of MegaDerm led to smaller amounts of drained serum and a shorter drain maintenance period than when AlloDerm was used. In contrast, no significant differences were found between CGDerm and AlloDerm, or between CGDerm and MegaDerm.

Keywords Acellular dermis, Mammoplasty, Reconstructive surgical procedures, Seroma

\section{INTRODUCTION}

With the increasing prevalence of breast cancer, mastectomy techniques have continuously improved to meet the exacting cosmetic

Received: Feb 8, 2017 Revised: Apr 4, 2017 Accepted: Apr 10, 2017 Correspondence: June-Kyu Kim Department of Plastic and Reconstructive Surgery, Kangbuk Samsung Medical Center, Sungkyunkwan University School of Medicine, 29 Saemunan-ro, Jongno-gu, Seoul 03181, Korea. E-mail: kokoro72@naver.com

Copyright @ 2017 The Korean Society for Aesthetic Plastic Surgery.

This is an Open Access article distributed under the terms of the Creative Commons Attribution Non-Commercial License (http://creativecommons.org/licenses/by-nc/4.0/) which permits unrestricted non-commercial use, distribution, and reproduction in any medium, provided the original work is properly cited. www.e-aaps.org needs of breast cancer patients. In patients with early-stage breast cancer, after a total skin-sparing mastectomy is conducted, the number of breast reconstructions using breast implants and acellular dermal matrix $(\mathrm{ADM})$ continues to increase [1].

Since the introduction of ADM in implant-based breast reconstruction in 2012, it has gained popularity because it has several advantages: ADM enables tissue coverage in the lower pole of the breast implant, it can correct implant malposition, and it can prevent tissue thickening and capsular contracture $[2,3]$.

However, despite these advantages, some studies have reported that ADM was strongly associated with postoperative complications. In particular, seroma formation has been actively studied. The incidence of seroma has been reported to range from $0.2 \%$ to $20 \%$ in prosthetic breast reconstruction. Seroma formation is asso- 
ciated with many problems. It inhibits wound healing, prolongs discomfort and increases the duration of hospital visits, and sometimes leads to subsequent complications such as infection, skin necrosis, and implant failure [1,3-7].

ADMs are categorized according to their source into heterogenic $\mathrm{ADM}$ from bovine and porcine sources, and allograft $\mathrm{ADM}$ from human cadaveric sources. Although it is known that donor screening, as well as the preparation and storage of each form of allograft $\mathrm{ADM}$, can result in different degrees of graft-taking by the body, it is not easy to study the mechanisms involved or to find published research about these mechanisms.

We performed direct-to-implant breast reconstructions using 3 types of human allograft products: CGDerm ${ }^{\circledR}$ (Daewoong Bio Corp., Seoul, Korea), AlloDerm ${ }^{\circledR}$ (LifeCell Corp., Branchburg, NJ, USA), and MegaDerm ${ }^{\circledR}$ (L\&C Bio Corp., Seongnam, Korea).

The purpose of this study was to obtain useful information for the selection of an ADM product by comparing the amount of collected serum and the maintenance period of the drain.

\section{METHODS}

This study was approved by the Institutional Review Board of our hospital. We conducted a retrospective study including 73 patients with breast cancer who received ADM-assisted, implant-based breast reconstruction after mastectomy from March 2011 to January 2016.

Patients' charts were reviewed to collect the following demographic characteristics: age, body mass index (BMI), breast reconstruction location, smoking history, axillary dissection, comorbidities (such as hypertension and diabetes mellitus [DM]), breast implant size, type and amount of ADM, the total amount of serum that was collected in the drain, and the maintenance period of the drain (Table 1).
Only patients who underwent allograft ADM-assisted direct-toimplant breast reconstruction were included in this study. The exclusion criteria were a rapid change in the amount of drained serum caused by complications (such as bleeding, hematoma, infection, or skin flap necrosis), and implant or ADM removal.

To reduce confounders that could affect postoperative serum formation, a single plastic surgeon participated in this study and performed operations using a single brand of breast implant ([MEN-

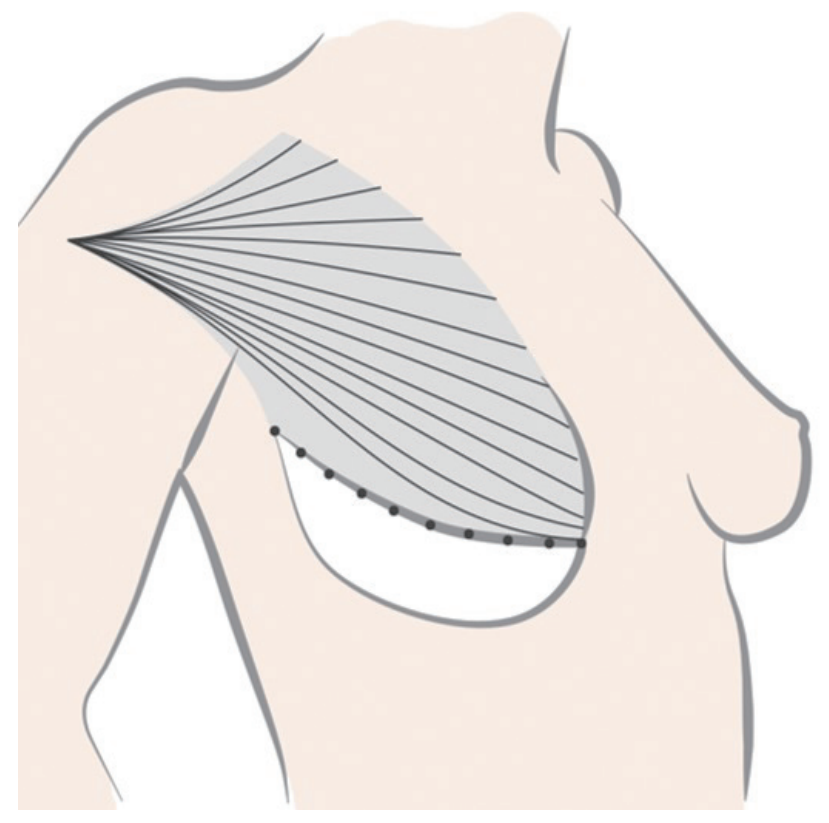

Fig. 1. Acellular dermal matrix (ADM) placement. Depiction of ADM placement. The selected ADM was fully hydrated with sterile normal saline and was sutured to the inferolateral border of pectoralis major muscle and the inframammary fold.

Table 1. Demographic and operative characteristics of the patients

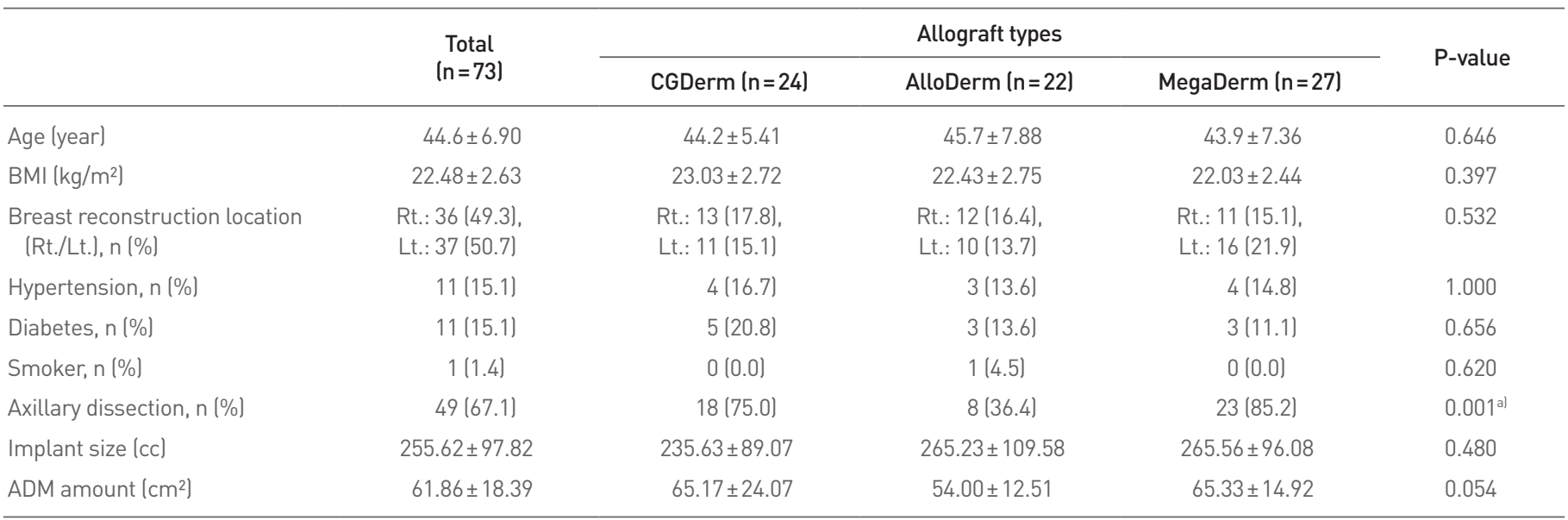

$B M I$, body mass index; Rt., right; Lt., left; ADM, acellular dermal matrix.

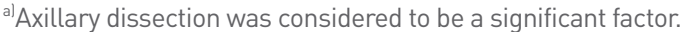


$\mathrm{TOR}^{\circledR}$; Mentor Worldwide LLC, Irvine, CA, USA] anatomical type), taking care to provide adequate hemostasis.

\section{Surgical technique}

After a total skin-sparing mastectomy was performed, the inferior border of the pectoralis major muscle was divided and dissected and a permanent breast implant fitting the breast measurements was inserted. The ADM was randomly selected from the 3 types of ADM (CGDerm, AlloDerm, and MegaDerm). The selected ADM was fully hydrated with sterile normal saline for 15 minutes. It was then sutured to the inferolateral border of the pectoralis major muscle and the inframammary fold (Fig. 1).

\section{Assessment of the amount of drained serum and the drain maintenance period}

Suction drains were inserted into the upper and lower poles of the submuscular plane. In the case of axillary dissection, an additional suction drain was inserted in the axillary area. The amount of serum that was collected in the drain was measured daily, and when it reached less than $30 \mathrm{cc} /$ day for 2 consecutive days, the drain was removed. The total amount of serum that was collected in the drain and the maintenance period of the drain were compared according to the type of ADM. We also checked for seroma formation after drain removal.

\section{Statistical analysis}

Patients' baseline characteristics were analyzed, and they were divided into 3 groups based on the type of ADM. To assess the correlation between ADM type and serum formation, axillary dissection, which was shown to be a significant variable in the univariate analysis, and factors assumed to be clinically relevant were selected as variables to adjust for. The chi-square test was used to analyze categorical variables, and analysis of variance was used to analyze continuous variables. Analysis of covariance was used to analyze differences in the mean values of the total amount of serum that was collected and the drain maintenance period across ADM types. All analyses were conducted using STATA (version 14; STATACorp, College Station, TX, USA), and P-values $<0.05$ were considered to indicate statistical significance.

\section{RESULTS}

\section{Patient demographics}

The mean age of the patients was 44.6 years (range, 23-61 years), and their mean BMI was $22.48 \mathrm{~kg} / \mathrm{m}^{2}$ (range, $17.13-29.62 \mathrm{~kg} / \mathrm{m}^{2}$ ). With regard to comorbidities, $15.1 \%$ and $15.1 \%$ of the patients had hypertension and DM, respectively. None of the patients received radiation therapy or chemotherapy during the preoperative period. The mean size of the implant was $255.62 \mathrm{cc}$ (range, 90-600 cc), the mean amount of ADM was $61.86 \mathrm{~cm}^{2}$ (range, $30-128 \mathrm{~cm}^{2}$ ) (Table 1), the mean total amount of serum drained was $880.37 \mathrm{cc}$ (range, $312-1,947 \mathrm{cc}$ ), and the mean maintenance period of the drain was 14.67 days (range, $5-23$ days).

Amount of drained serum and drain maintenance period An analysis of the total amount of drained serum and the maintenance period according to ADM type revealed the following: 1) the average amount of drained serum with CGDerm was $896.95 \pm 317.37$ cc $(95 \%$ CI, 762.94-1,030.97 cc) and the drain was removed after $15.00 \pm 3.68$ days (95\% CI, 13.44-16.56 days); 2 ) for AlloDerm, the average amount of drained serum was 1,060.38 $\pm 483.97 \mathrm{cc}(95 \%$ CI, $845.80-1,274.96 \mathrm{cc}$ ) and the drain was removed after an average of $16.50 \pm 4.50$ days ( $95 \%$ CI, $14.50-18.50$ days); and 3 ) the average amount of drained serum for MegaDerm was $718.94 \pm 275.04 \mathrm{cc}$ ( $95 \%$ CI, 610.14-827.75 cc), and the drain was removed after 12.89 \pm 3.02 days ( $95 \%$ CI, $11.70-14.08$ days) (Table 2). A total of 19 cas-

Table 2. Comparison of the 3 types of ADM

\begin{tabular}{|c|c|c|c|c|c|}
\hline & \multicolumn{3}{|c|}{ Allograft types } & \multirow{2}{*}{ P-value } & \multirow{2}{*}{$\begin{array}{l}\text { Adjusted } \\
\text { P-value }^{\text {al }}\end{array}$} \\
\hline & CGDerm & AlloDerm & MegaDerm & & \\
\hline Total amount drained (cc) & $896.95 \pm 317.37$ & $1,060.38 \pm 483.97$ & $718.94 \pm 275.04$ & 0.007 & 0.009 \\
\hline Drain maintenance period (day) & $15.00 \pm 3.68$ & $16.50 \pm 4.50$ & $12.89 \pm 3.02$ & 0.005 & 0.022 \\
\hline
\end{tabular}

ADM, acellular dermal matrix.

a)Adjusted for patients' age, diabetes mellitus, body mass index, axillary dissection, amount of ADM, and implant size.

Table 3. Seroma formation

\begin{tabular}{|c|c|c|c|c|c|}
\hline & \multirow{2}{*}{$\begin{array}{c}\text { Total } \\
\text { (n=73) }\end{array}$} & \multicolumn{3}{|c|}{ Allograft types } & \multirow{2}{*}{ P-value } \\
\hline & & CGDerm (n=24) & AlloDerm ( $n=22$ ) & MegaDerm ( $n=27)$ & \\
\hline Seroma formation (\%) & $19(26.0)$ & $6(8.22)$ & 7 (9.59) & $6(8.22)$ & 0.741 \\
\hline
\end{tabular}

There was no significant difference in seroma formation among the allograft groups. 


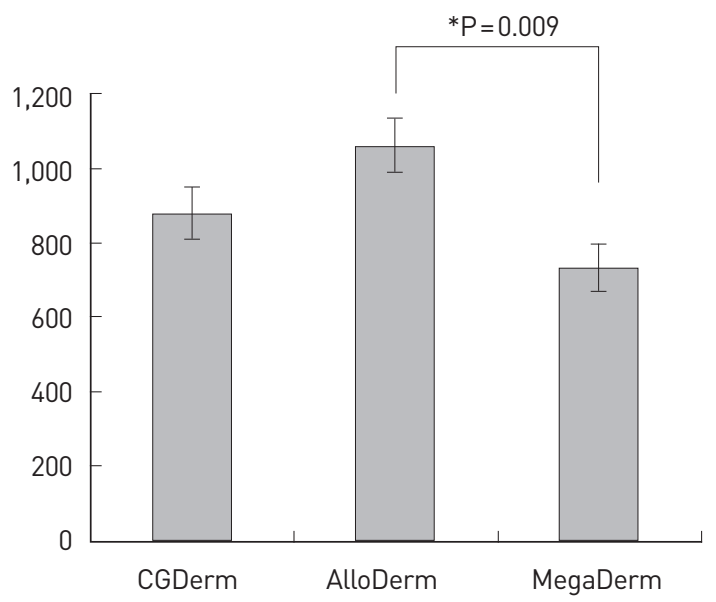

Fig. 2. Total amount of drained serum. The mean values (standard error [SE]) of the total drained amounts according to the type of acellular dermal matrix.

es of seroma formation (26\%) were observed after drain removal. No significant differences in seroma formation were found among the ADM groups $(\mathrm{P}=0.741)$ (Table 3$)$.

In addition to axillary dissection, which was found to be a significant variable $(\mathrm{P}=0.001)$ in the univariate analysis, the existing literature suggests that factors such as age, BMI, DM, the amount of $\mathrm{ADM}$, and implant size are closely related to seroma formation [1,8-11]. Our results remained significant after adjusting for these factors. Compared to AlloDerm, MegaDerm showed a significantly smaller total drained amount $(\mathrm{P}=0.009)$, and a significantly shorter drain maintenance period $(\mathrm{P}=0.022)$. However, no significant differences were found between CGDerm and AlloDerm or between CGDerm and MegaDerm (Fig. 2 and 3).

\section{DISCUSSION}

In general, breast reconstruction using ADM has many advantages including 1) maintenance of the inframammary fold and a more natural projection of the lower pole; 2) covering the lower lateral position of the breast implant and securing its position; 3 ) less dissection of the surrounding tissues such as the pectoralis major muscle and serratus anterior muscle, ultimately contributing to a reduction in the damage to these tissues; and 4) reduction in the tension of the overlying skin flap and the provision of assistance in the correction and treatment of capsular contracture [2,3,8-10].

However, some studies have reported that ADM could increase the risk of infection and the occurrence of seroma. Chun et al. [1] reported that the use of ADM increased the risk of seroma occurrence by 4.24 times, and of infection by 5.37 times, whereas Parks et al. [11] reported that it increased the risk of seroma by almost 2 times. Furthermore, Jordan et al. [7] reported that such an increase in the occurrence of seroma multiplied the chance of tissue expand-

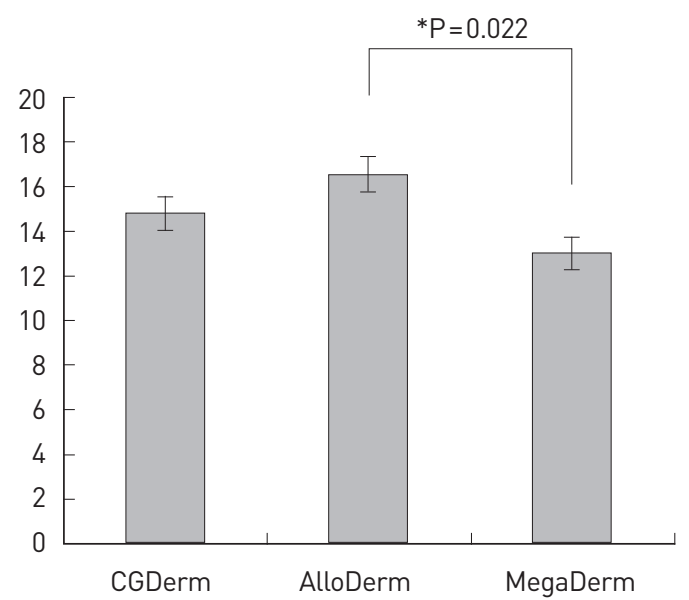

Fig. 3. Maintenance period of the drain. The mean values (standard error [SE]) of the drain maintenance period according to the type of acellular dermal matrix.

er removal by 4.42 times and of infection-induced tissue expander removal by 6.71 times [12]. However, other studies reported no significant association between the use of ADM and seroma formation $[13,14]$.

As such, although studies related to the use of ADM and the occurrence of complications have been actively carried out, insufficient research has been conducted into the stability of different ADM types. We thought that evaluating the volume of drained serum would be a useful method to compare ADM stability immediately after surgery. For this reason, we collected the serum generated immediately after surgery to measure its total amount and the drain maintenance period. We postulated that less serum and a shorter maintenance period indicate more stable engraftment, so the amount of serum drained with each ADM was compared. Among the different ADMs, AlloDerm had the greatest mean drained amount and MegaDerm had the lowest; the differences between MegaDerm and AlloDerm were significant.

In addition, it is important to determine the appropriate timing for removing the drain. If a drain is hastily removed before drainage decreases adequately, seroma can occur $[7,11,15,16]$. Few studies have reported the exact removal time of the drain postoperatively. Based on seroma formation, many studies have suggested that the optimal timing for removing the drain is when less than $30 \mathrm{cc}$ per day of serum is collected for 2 consecutive days. We also removed drains based on this criterion $[1,10,12,17,18]$. AlloDerm had the longest drain maintenance period, while MegaDerm had the shortest. As was the case with the amount of drained serum, the difference between MegaDerm and AlloDerm (3.8 days, $\mathrm{P}=0.005$ ) was significant.

We experienced a total of 19 cases of seroma formation (26\%) after drain removal (Table 3). Patients clinically presenting with seroma underwent simple aspiration. As a prophylactic antibiotic, 
$1,000 \mathrm{mg}$ of cephalexin was prescribed 3 times a day for 1 week, and patients were asked to visit the outpatient clinic every 2 days $[7,19]$. In almost all patients, the seroma resolved with less than 3 aspirations. One patient in the AlloDerm group experienced seroma recurrence after 3 aspirations and a superimposed infection was suspected, as erythematous changes and a heating sensation were observed in the lower pole of the breast. Resolution was obtained after 3 more aspirations were performed every 2 days and an intravenous antibiotic (cefminox) was administered for 2 weeks.

It is known that the degree of ADM engraftment can affect the level of inflammation, vascular permeability, encapsulation, and fibrosis. The difference in the degree of engraftment can be explained by the variability in processing and storing methods of the $3 \mathrm{ADM}$ types $[4,17,19,20]$.

We hypothesized that there would be differences in the amount of serum formation and the drain maintenance period, because differences exist in the processing and storing methods, even within the same groups of allograft ADMs. MegaDerm is characterized by an electron beam irradiation process, which involves ionizing radiation. The cross-linking density of the collagen matrix, the main element of $\mathrm{ADM}$, is high due to this electron beam irradiation. As a result, host cell infiltration rapidly develops into the implanted $\mathrm{ADM}$, and extracellular matrix expression and microvessel formation are also increased [21]. Therefore, it can be assumed that adequate host integration leads to engraftment and less serum formation.

In conclusion, MegaDerm showed less drained serum and a shorter maintenance period of the drain than AlloDerm. As there were no significant differences between CGDerm and AlloDerm, or between CGDerm and MegaDerm, surgeons can make an arbitrary decision between these types based on their clinical experience.

\section{PATIENT CONSENT}

Patients provided written consent for the use of their images.

\section{REFERENCES}

1. Chun YS, Verma K, Rosen H, et al. Implant-based breast reconstruction using acellular dermal matrix and the risk of postoperative complications. Plast Reconstr Surg 2010;125:429-36.

2. Duncan DI. Correction of implant rippling using allograft dermis. Aesthet Surg J 2001;21:81-4.

3. Sbitany H, Serletti JM. Acellular dermis-assisted prosthetic breast reconstruction: a systematic and critical review of efficacy and associated morbidity. Plast Reconstr Surg 2011;128:1162-9.

4. Hoppe IC, Yueh JH, Wei CH, et al. Complications following expander/implant breast reconstruction utilizing acellular dermal matrix: a systematic review and meta-analysis. Eplasty 2011;11:e40.

5. Caputo GG, Franchini Z, Maritan M, et al. Daily serum collection after acellular dermal matrix-assisted breast reconstruction. Arch Plast
Surg 2015;42:321-6.

6. Israeli R. Complications of acellular dermal matrices in breast surgery. Plast Reconstr Surg 2012;130:159s-72s.

7. Jordan SW, Khavanin N, Kim JY. Seroma in prosthetic breast reconstruction. Plast Reconstr Surg 2016;137:1104-16.

8. Breuing $\mathrm{KH}$, Warren SM. Immediate bilateral breast reconstruction with implants and inferolateral AlloDerm slings. Ann Plast Surg 2005; 55:232-9.

9. Breuing KH, Colwell AS. Inferolateral AlloDerm hammock for implant coverage in breast reconstruction. Ann Plast Surg 2007;59:250-5.

10. Neaman KC, Armstrong SD, Baca ME, et al. Outcomes of traditional cosmetic abdominoplasty in a community setting: a retrospective analysis of 1008 patients. Plast Reconstr Surg 2013;131:403e-10e.

11. Parks JW, Hammond SE, Walsh WA, et al. Human acellular dermis versus no acellular dermis in tissue expansion breast reconstruction. Plast Reconstr Surg 2012;130:739-46.

12. Mendenhall SD, Anderson LA, Ying J, et al. The BREASTrial: stage I. Outcomes from the time of tissue expander and acellular dermal matrix placement to definitive reconstruction. Plast Reconstr Surg 2015; 135:29e-42e.

13. Lanier ST, Wang ED, Chen JJ, et al. The effect of acellular dermal matrix use on complication rates in tissue expander/implant breast reconstruction. Ann Plast Surg 2010;64:674-8.

14. Colwell AS, Damjanovic B, Zahedi B, et al. Retrospective review of 331 consecutive immediate single-stage implant reconstructions with acellular dermal matrix: indications, complications, trends, and costs. Plast Reconstr Surg 2011;128:1170-8.

15. Woerdeman LA, Hage JJ, Smeulders MJ, et al. Skin-sparing mastectomy and immediate breast reconstruction by use of implants: an assessment of risk factors for complications and cancer control in 120 patients. Plast Reconstr Surg 2006;118:321-30.

16. Weichman KE, Wilson SC, Saadeh PB, et al. Sterile "ready-to-use" AlloDerm decreases postoperative infectious complications in patients undergoing immediate implant-based breast reconstruction with acellular dermal matrix. Plast Reconstr Surg 2013;132:725-36.

17. Glasberg SB, Light D. AlloDerm and Strattice in breast reconstruction: a comparison and techniques for optimizing outcomes. Plast Reconstr Surg 2012;129:1223-33.

18. Moyer KE, Potochny JD. Technique for seroma drainage in implantbased breast reconstruction. J Plast Reconstr Aesthet Surg 2012;65: 1614-7.

19. Michelotti BF, Brooke S, Mesa J, et al. Analysis of clinically significant seroma formation in breast reconstruction using acellular dermal grafts. Ann Plast Surg 2013;71:274-7.

20. Yuen JC, Yue CJ, Erickson SW, et al. Comparison between freeze-dried and ready-to-use AlloDerm in alloplastic breast reconstruction. Plast Reconstr Surg Glob Open 2014;2:e119.

21. Lee JH, Kim HG, Lee WJ. Characterization and tissue incorporation of cross-linked human acellular dermal matrix. Biomaterials 2015;44: 195-205. 